\title{
Is There a Limit to the Superiority of Individuals with ASD in Visual Search?
}

\author{
Roy S. Hessels • Ignace T. C. Hooge • \\ Tineke M. Snijders $\cdot$ Chantal Kemner
}

Published online: 10 July 2013

(c) Springer Science+Business Media New York 2013

\begin{abstract}
Superiority in visual search for individuals diagnosed with autism spectrum disorder (ASD) is a wellreported finding. We administered two visual search tasks to individuals with ASD and matched controls. One showed no difference between the groups, and one did show the expected superior performance for individuals with ASD. These results offer an explanation, formulated in terms of load theory. We suggest that there is a limit to the superiority in visual search for individuals with ASD, related to the perceptual load of the stimuli. When perceptual load becomes so high that no additional task-
\end{abstract}

R. S. Hessels - I. T. C. Hooge - T. M. Snijders - C. Kemner Department of Experimental Psychology, Helmholtz Institute, Utrecht University, Utrecht, The Netherlands

R. S. Hessels · T. M. Snijders · C. Kemner

Department of Developmental Psychology, Utrecht University, Utrecht, The Netherlands

R. S. Hessels ( $\bowtie) \cdot$ I. T. C. Hooge · C. Kemner

Heidelberglaan 2, 3584 CS Utrecht, The Netherlands

e-mail: royhessels@gmail.com

T. M. Snijders · C. Kemner

Department of Psychiatry, Brain Center Rudolf Magnus,

University Medical Centre, Heidelberglaan 100,

3584 CX Utrecht, The Netherlands

Present Address:

T. M. Snijders

Centre for Language Studies, Radboud University Nijmegen,

Nijmegen, The Netherlands

\section{T. M. Snijders}

Donders Institute for Brain, Cognition and Behaviour,

Radboud University Nijmegen, Nijmegen, The Netherlands

T. M. Snijders

Kapittelweg 29, 6525 EN Nijmegen, The Netherlands (ir)relevant information can be processed, performance will be based on single stimulus identification, in which no differences between individuals with ASD and controls have been demonstrated.

Keywords Autism spectrum disorder · Visual search · Superiority $\cdot$ Perceptual load $\cdot$ Load theory

Individuals who have been diagnosed with autism spectrum disorder (ASD) typically exhibit impairments in social interaction and communication, whilst on the other hand excel on certain visuospatial tasks (see Dakin and Frith 2005; Simmons et al. 2009 for an overview). This is possibly highly beneficial for early recognition of ASD, and indeed, studies on processing of eye-gaze and visual fixation during social interaction in infancy have already been successful in distinguishing infants at-risk for autism from controls (Elsabbagh et al. 2009; Merin et al. 2006). Nonsocial visuospatial differences might prove to be the next step in early recognition of ASD. However, such prospects are only viable if the perceptual differences between individuals with ASD and typically developing individuals are well understood.

A particularly well-documented finding is the excellence of individuals with ASD in visual search (Jarrold et al. 2005; Joseph et al. 2009; Kemner et al. 2007; O'Riordan 2004; O'Riordan et al. 2001; Plaisted et al. 1998a), where individuals with ASD show shorter reaction times in visual search tasks when compared to a matched control group. This excellence manifests itself even as early as in 2.5-year old toddlers (Kaldy et al. 2011). In this study, eye-movements of toddlers were measured and toddlers with ASD proved more successful at finding a target distinct by a combination of features (Kaldy et al. 2011). A possible 
explanation for these findings is an enhanced stimulus discriminability in individuals with ASD, which is demonstrated by increasing differences between groups in reaction time when the task becomes more difficult (O'Riordan et al. 2001; Plaisted et al. 1998a). This is also supported by the finding that autistic children outperform the control group in an embedded figures tasks where the target is embedded in a more complex image (Jarrold et al. 2005). This superiority in visual search also seems to translate to the typically developing population, where individuals high on the autistic traits spectrum, as measured with the Autism Spectrum Quotient (Baron-Cohen et al. 2001), outperform individuals low on the autistic traits spectrum in a visual search task (Almeida et al. 2010).

Another explanation might be that individuals with ASD adopt a different search strategy, and are more successful or faster because of this. To test whether different search strategies between individuals with ASD and controls might explain the enhanced performance of individuals with ASD, several studies looked at eye-movements during visual search (Joseph et al. 2009; Kemner et al. 2007). Kemner et al. (2007) conclude that individuals with ASD do not adopt a more efficient search strategy, which would be accompanied by longer fixation times (Hooge and Erkelens 1999), and that the superior performance might thus be due to enhanced stimulus discrimination. In addition, Joseph et al. (2009) conclude that participants with ASD search the displays in a similar fashion as controls, and that the superior performance is due to nonsearch processes, i.e. stimulus discrimination, not searchstrategy. Furthermore, children with ASD do not appear to search efficiently in a more ecological search setting (Pellicano et al. 2011).

The literature seems to provide a general consensus on superior performance for individuals with ASD in visual search, likely due to enhanced stimulus discrimination, which becomes more pronounced when task difficulty increases. This superior performance is evident from reduced reaction times in individuals with ASD, while error rates are equal to control participants. As this superior performance is well replicated, the present study aimed to use this as a baseline for comparison with effects between individuals with ASD and controls in parallel studies. A visual search task using a vertical target among tilted distractors was used to accomplish this. However, result from this experiment did not show the expected superiority for individuals with ASD compared to the control group. This incongruence with the literature led the authors to do a second experiment in which the search superiority for individuals with ASD was again demonstrated. The results will be discussed and put in perspective with current theories on enhanced stimulus discrimination in ASD.

\section{Method Experiment 1}

\section{Participants}

19 young adults with ASD (4 female, 15 male) and 31 matched control subjects ( 7 female, 24 male) participated in experiment 1. Mean age of the ASD group was 21.6 $(\mathrm{SD}=3.04)$. Mean age of the control group was 21.6 $(\mathrm{SD}=2.11)$. For the ASD group, the Wechsler Adult Intelligence Scale III, Dutch edition (WAIS-III), was used to determine IQ scores. For the control group the Wechsler Abbreviated Scale of Intelligence (WASI) was used to estimate IQ. The Freiburg Visual Acuity Test (Bach 1996) was used to measure visual acuity and Weber contrast sensitivity (i.e. sensitivity for contrast between feature and background luminance). The ASD and control groups did not differ significantly on age, IQ, visual acuity or contrast sensitivity, and all subjects had normal or corrected-tonormal vision (log MAR acuity range -0.49 to 0.35 ). The Autism Spectrum Quotient (AQ; Baron-Cohen et al., 2001) was administered to all subjects. Due to technical reasons AQ data of two control subjects was lost. Mean AQ for the patient group was $32.7(\mathrm{SD}=4.16)$ and $24.4(\mathrm{SD}=3.22)$ for the control group. An independent $t$ test was used to determine significance, $t(46)=7.86, p<.001$. Descriptive statistics for both groups are given in Table 1 .

The diagnostic evaluation for the ASD group included a psychiatric observation and a review of prior records (developmental history, child psychiatric and psychological observations and tests). ASD was diagnosed by a child psychiatrist using the DSM-IV criteria. The ASD group consisted of 12 participants diagnosed with Asperger's Syndrome, 6 diagnosed with Autism, and 1 diagnosed with PDD-NOS. The parents of all but one of these subjects were administered the Autism Diagnostic Interview (Lord et al. 1994) and eleven of the participants with ASD were administered the Autism Diagnostic Observation Schedulegeneric (Lord et al. 1989), both by a trained rater. Seventeen subjects met ADI-R criteria for autism or autism spectrum disorder; one subject did not (this subject did, however, meet DSM-IV criteria). All of the participants

Table 1 Means and standard deviations for age, IQ, AQ, contrast sensitivity and visual acuity for the ASD and the matched control group

\begin{tabular}{lll}
\hline & Control group & ASD group \\
\hline Sample size & 31 & 19 \\
Age & $21.6(2.11)$ & $21.6(3.04)$ \\
IQ & $118.9(11.1)$ & $116.2(12.2)$ \\
AQ & $24.4(3.22)$ & $32.7(4.16)$ \\
Weber contrast sensitivity & $1.69 \%(1.32 \%)$ & $1.33 \%(0.58 \%)$ \\
Visual acuity (log MAR) & $-0.24(0.16)$ & $-0.25(0.11)$ \\
\hline
\end{tabular}


who completed the ADOS-G met the full criteria for autism or autism spectrum disorder.

Control subjects were recruited on campus and were screened for ASD, ASD in their family, and history of psychopathology. None of the control subjects reported ASD or psychopathology, although two subjects had firstdegree relatives diagnosed with ASD.

Both the subjects with ASD and the control participants received a money reward for their participation. The study was approved by the medical ethics committee of the University Medical Centre Utrecht and subjects gave written informed consent prior to participation.

\section{Stimuli and Task}

Search displays consisted of a dark-grey background containing 25 light-grey line elements (see Fig. 1).

In the 'absent' condition, all lines were tilted $10^{\circ}$ clockwise. In the 'present' condition one line element (i.e. the target) was vertical, whilst the remaining 24 lines were again tilted $10^{\circ}$ clockwise. 50 'absent' and 50 'present' trials were presented in a pseudorandom order. Participants performed a binary forced choice task indicating whether the target was absent or present. Each trial started with a fixation cross. After the subject pressed the space bar, the search display appeared with a stimulus-onset asynchrony of 0-1,000 ms. The search display remained on screen until the subject pressed one of the response keys. Response keys were the left and the right arrow-keys on the keyboard. The keys representing target absent and target present were counterbalanced across participants. Response on each trial initiated the fixation phase of the next trial.

\section{Procedure}

Participants were seated in a height-adjustable chair at $57 \mathrm{~cm}$ distance from a $22^{\prime \prime}$ monitor. A chin-rest was used to

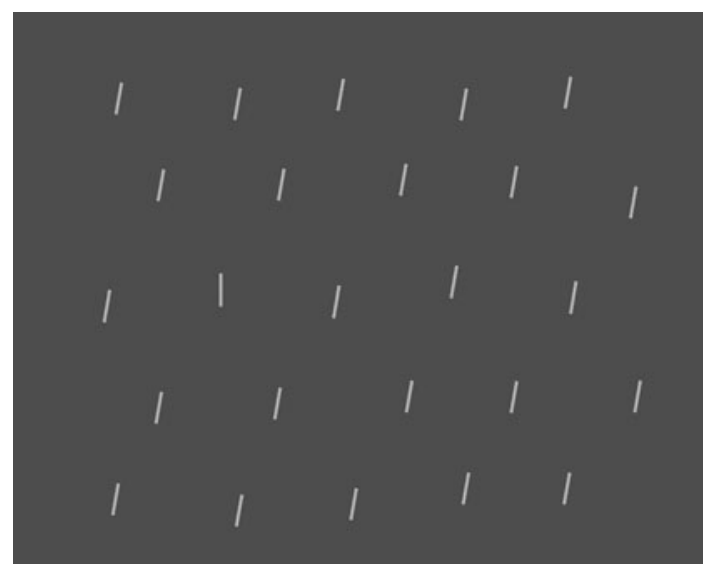

Fig. 1 Example stimulus in target 'present' condition maintain viewing distance. The size of the search displays was $31.25^{\circ} \times 25^{\circ}$. A black curtain was then drawn over the participant and the screen so that no external light influenced the setup.

\section{Results Experiment 1}

Mean reaction times and error rates were calculated for target present and target absent trials in both groups. One of the clinical subjects was eliminated from the analysis, as mean reaction time for target absent trials for this participant was $10.3 \mathrm{~s}$ compared to a mean reaction time of the other young ASD adults of $2.77 \mathrm{~s}(\mathrm{SD}=1.03 \mathrm{~s})$. Other than that, no trials were excluded from the analysis.

\section{RT Analysis}

A repeated measures analysis of variance (ANOVA) with target (i.e. present or absent) as a within-subjects factor and group (i.e. ASD or control) as a between-subjects factor was used to determine statistical significance. The ANOVA revealed a significant effect of target on reaction time $\left(\mathrm{F}(1,47)=89.22, p<.0005, \eta_{\mathrm{p}}^{2}=0.66\right)$, reflecting the fact that both groups were faster on target present trials as compared to target absent trials. No differences were seen between the ASD group and controls (see Fig. 2). There was no effect of group $(\mathrm{F}<1)$, nor was there an interaction between target and group $(\mathrm{F}<1)$. The results are depicted in Fig. 2.

\section{Error Analysis}

A repeated measures ANOVA revealed a significant effect of target on error rate $\left(\mathrm{F}(1,47)=49.13, p<.0005, \eta_{\mathrm{p}}^{2}=0.51\right)$, indicating that the error rate in the target present condition $(\mathrm{m}=0.142, \mathrm{SD}=0.125)$ was significantly higher than in the target absent condition $(\mathrm{m}=0.007, \mathrm{SD}=0.013)$. Again, there was no effect of group $(\mathrm{F}<1)$, nor was there an interaction between target and group $(\mathrm{F}<1)$.

\section{Discussion Experiment 1}

The purpose of experiment 1 was to replicate the general finding that the ASD group shows superior performance in visual search. To accomplish this a visual search task was administered in which participants searched for a vertical target among tilted distractors. Both the ASD group and the control group were significantly slower to react when the target was absent, consistent with general findings in visual search. There was, however, no superior performance for the ASD group, neither when the target was present nor absent. More errors were made by both groups when the 


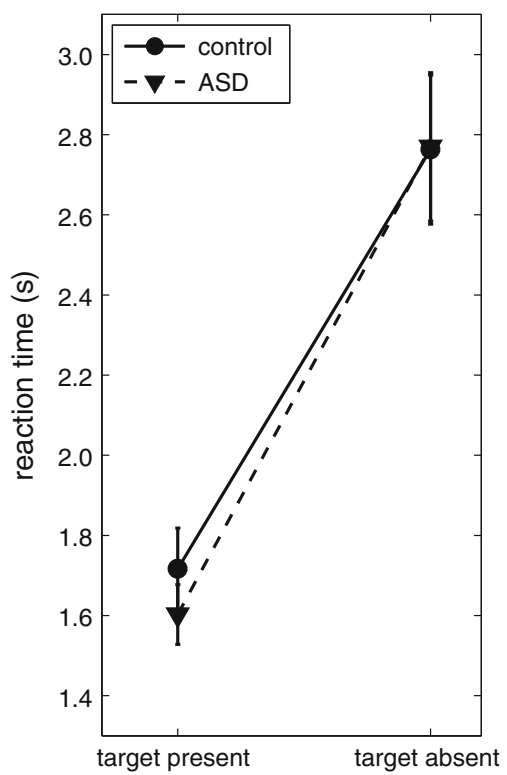

Fig. 2 Mean reaction times with SE for target absent and target present trials in ASD and controls

target was present compared to target absent trials. However, no differences in error rates between the ASD group and the control group were identified, neither when the target was present nor when it was absent.

These results are intriguing, and in high contrast with the body of research on visual search in ASD. The study by Kemner et al. (2007) used similar stimuli and task as the present study, but, in contrast to the present study, did show clear differences in visual search performance between subjects with ASD and controls. The only differences between the two experiments were that the present experiment consisted of only one set size (i.e. 25 elements), and that the distractors were slightly less tilted from vertical (i.e. $10^{\circ}$ clockwise tilt in the present experiment compared to a $17^{\circ}$ clockwise tilt in Kemner et al. 2007). Furthermore, the groups in both studies were very similar regarding age, IQ and diagnosis. Finally, it should be noted that in the earlier study by Kemner et al. (2007), the effect between the ASD group and controls was numerically largest in the display resembling the present experiment the most; the 25 -element display with a vertical target between $17^{\circ}$ tilted distractors. To resolve this apparent incongruence, a second experiment was conducted with stimuli constructed to match those in Kemner et al. (2007).

\section{Method Experiment 2}

Participants

13 young adults with ASD and 14 matched control subjects participated in experiment 2.12 control participants and 12 participants with ASD had previously participated in experiment 1. AQ scores for these participants were compared using an independent-samples $t$ test, $t(22)=4.95$, $p<.001$. At least 6 months had passed between experiment 1 and 2. For the 2 control participants who had not participated in experiment 1, the Wechsler Abbreviated Scale of Intelligence was used to estimate IQ. To the young ASD adult who had not participated before the full Wechsler Adult Intelligence Scale was administered. This participant was diagnosed with Autism according to DSMIV criteria, resulting in a group total of 7 diagnosed with Autism. Furthermore, the group comprised 5 participants diagnosed with Asperger's Syndrome and 1 participant diagnosed with PDD-NOS. The ASD group and the matched control group did not differ significantly on age, IQ, visual acuity or contrast sensitivity. Descriptive statistics are provided in Table 2.

Stimuli and Task

The experiment consisted of two sessions, one 'easy' and one 'hard' session (i.e. analogous to Kemner et al. 2007), which were counterbalanced across participants. Stimuli again consisted of dark-grey displays containing light-grey line elements. The set-size of lines in the stimulus display was varied in both sessions with 4,16 or 25 lines in the display. In the 'easy' session, the display consisted of vertical line distractors with a target line tilted $17^{\circ}$ clockwise in half of the displays. In the 'hard' sessions, the display consisted of $17^{\circ}$ clockwise tilted line distractors, with a vertical line target in half of the displays. 30 trials were presented per condition, resulting in a total of 180 trials [30 trials $* 2$ (target present or absent) $* 3$ (set-size)] per session. Participants performed a binary forced choice task indicating whether the target was absent or present. Each trial started with a fixation cross. After the subject pressed the space bar, the search display appeared immediately. The search display remained on screen until the subject pressed one of the response keys. Participants responded with the left and the right arrow-keys on the keyboard. The left arrow key represented the vertical line,

Table 2 Means and standard deviations for age, IQ, AQ, contrast sensitivity and visual acuity for the ASD and matched control group

\begin{tabular}{lll}
\hline & Control group & ASD group \\
\hline Sample size & 14 & 13 \\
Age & $22.8(2.46)$ & $22.9(4.05)$ \\
IQ & $118.9(13.5)$ & $116.1(11.6)$ \\
AQ & $24.7(3.58)$ & $32.4(4.08)$ \\
Weber contrast sensitivity & $1.27 \%(0.34 \%)$ & $1.54 \%(0.42 \%)$ \\
Visual acuity (log MAR) & $-0.26(0.12)$ & $-0.25(0.11)$ \\
\hline
\end{tabular}


and was to be pressed when target was present in the 'hard' session and when the target was absent in the 'easy' session. The right arrow key represented the tilted line and was to be pressed when the target was present in the 'easy' session and when the target was absent in the 'hard' session. The response on each trial initiated the fixation phase of the next trial.

\section{Procedure}

The procedure was identical to experiment 1 .

\section{Results Experiment 2}

Mean reaction times and error rates were calculated for target absent and target present trials in all set sizes. Analyses were done for the 'easy' and 'hard' session separately. No trials were excluded from the analysis.

\section{RT Analysis}

A repeated measures ANOVA with Greenhouse-Geisser correction using target (absent or present) and set size (4, 16 or 25) as within subjects factors and group (ASD or control) as a between subjects factor was used to determine statistical significance. The results are depicted in Fig. 3.

In the 'easy' session, a main effect for target was observed $\left(\mathrm{F}(1,25)=18.96, p<.0005, \eta_{\mathrm{p}}^{2}=0.43\right)$, reflecting longer reaction times when the target was absent (609 ms for target present vs. $740 \mathrm{~ms}$ for target absent). Reaction times increased with set size $(\mathrm{F}(1.29,32.21)=7.50, p<.01$, $\eta_{\mathrm{p}}^{2}=0.23$, with mean reaction times of 644,682 and $697 \mathrm{~ms}$, respectively. A significant two-way interaction between target and set size $(\mathrm{F}(1.12,28.04)=9.01, p<.005$, $\left.\eta_{\mathrm{p}}^{2}=0.27\right)$ reflected the rise in reaction time due to set size especially when the target was absent.

As can be seen in Fig. 3, clear differences between the ASD and control group were found. The between subjects effect for group was significant $(\mathrm{F}(1,25)=4.25, p=.05$, $\left.\eta_{\mathrm{p}}^{2}=0.15\right)$, indicating the overall reduction in reaction time for the ASD group compared to the control group. The two-way interaction between target and group $(\mathrm{F}(1,25)=$ $\left.5.86, p<.05, \eta_{\mathrm{p}}^{2}=0.19\right)$ reflected the difference between groups especially for the target absent condition. Finally, the three-way interaction of target, set size and group was significant $\left(\mathrm{F}(1.12,28.04)=4.53, p<.05, \eta_{\mathrm{p}}^{2}=0.15\right)$ and reflects the increase in reaction times due to set size when the target was absent, particularly for the control group.

In the 'hard' session, again longer reaction times were found for target absent trials $\left(\mathrm{F}(1,25)=12.74, p<.005, \eta_{\mathrm{p}}^{2}=0.34\right)$; $865 \mathrm{~ms}$ for target present vs. $1062 \mathrm{~ms}$ for target absent. Reaction times increased with set size $(\mathrm{F}(1.08,27.03)=8.33$, $\left.p<.01, \eta_{\mathrm{p}}^{2}=0.25\right)$; with mean reaction times of $856 \mathrm{~ms}$, $1011 \mathrm{~ms}$ and $1023 \mathrm{~ms}$, respectively. A significant target and set size interaction was observed $(\mathrm{F}(1.39,34.86)=12.02$, $\left.p<.0005, \eta_{\mathrm{p}}^{2}=0.33\right)$. Although the pattern of results in the 'hard' session resembled that in the 'easy' session (see Fig. 3), the main between subjects effect for group $(\mathrm{F}(1,25)=2.04$, $\left.p=.17, \quad \eta_{\mathrm{p}}^{2}=0.08\right)$, the target and group interaction $\left(\mathrm{F}(1,25)=2.42, p=.13, \eta_{\mathrm{p}}^{2}=0.09\right)$, the set size and group interaction $\left(\mathrm{F}(1.08,27.03)=1.01, p=.33, \eta_{\mathrm{p}}^{2}=0.04\right)$ and
Fig. 3 Mean reaction times with SE. Left graph depicts reaction time at set sizes 4,16 and 25 for ASD and control groups when the target was present. Right graph depicts reaction time when the target was absent

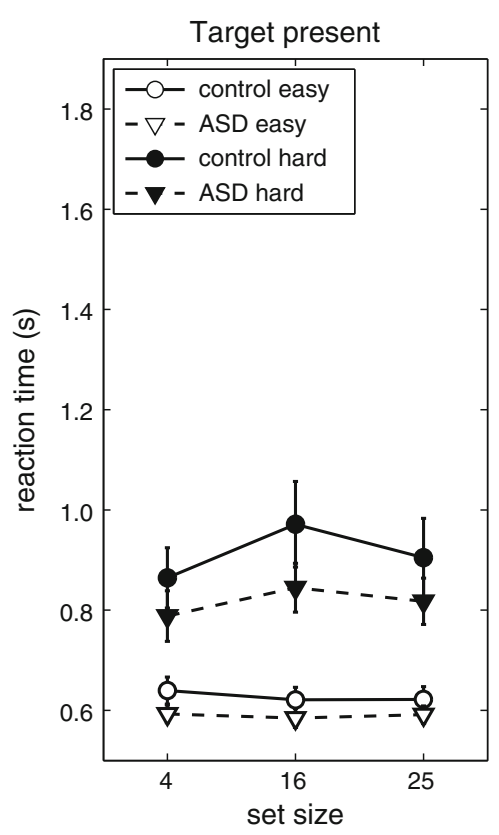


the target, set size and group three-way interaction $\left(\mathrm{F}(1.39,34.86)=1.88, p=.18, \eta_{\mathrm{p}}^{2}=0.07\right)$ were not significant for the 'hard' condition.

\section{Error Analysis}

Mean error rates in the easy session were 0.025 $(\mathrm{SD}=0.007)$ in the target present condition and 0.016 $(\mathrm{SD}=0.003)$ in the target absent condition. A repeated measures ANOVA on error rates in the easy session revealed that these means did not differ significantly, nor were any other significant main or interaction effects observed.

A repeated measures ANOVA on error rates in the hard session revealed a significant main effect for target $(\mathrm{F}(1,25)=10.23, p<.005)$, indicating that the error rate in the target present condition $(\mathrm{m}=0.044, \mathrm{SD}=0.008)$ was significantly higher than in the target absent condition $(\mathrm{m}=0.015, \mathrm{SD}=0.003)$. No main between subjects effect for group was observed, nor were there interactions involving the group factor.

\section{General Discussion}

The aim of present study was to replicate the general finding of superiority in visual search for a clinical group diagnosed with ASD. Furthermore, the aim was to establish a baseline of superior performance in visual search to be compared with intergroup effects in parallel studies. However, the results of experiment 1 indicated no such superior performance for the ASD group compared to matched controls. To uncover why this difference in performance was absent, a second experiment was conducted more closely resembling the Kemner et al. (2007) study. A visual search display varying in set size (i.e. 4,16 or 25 elements) was used, and the experiment consisted of two separate sessions. In one session the target was a tilted line among vertical line distractors, referred to as the 'easy' session, and in the other session the target was a vertical line among tilted line distractors, referred to as the 'hard' session. The naming of these sessions (i.e. 'easy' or 'hard') is in accordance with earlier research using such visual search displays (Kemner et al. 2007; O'Riordan et al. 2001). All but one individual with ASD and two controls had previously participated in the first experiment.

Results from the second experiment were in accordance with general findings in visual search literature. Reaction times increased when the target was absent, and reaction times also increased with set size, particularly when the target was absent. As in the Kemner et al. (2007) study, in the 'easy' session, the ASD group was significantly faster than their matched controls, especially when the target was absent. Furthermore, this difference between the ASD group and the control when the target was absent increased as set size became larger. In the 'hard' session, however, the superior performance for the ASD group was not significant. Why this might be the case will be discussed later on. For now, the results from experiment 1 indicate no differences between the ASD group and the control group, whereas the results from experiment 2 do show a superior performance for the ASD group compared to the control group.

In order to put these results in context, we have to consider the differences between experiment 1 and 2 . Experiment 2 was set up to match the Kemner et al. (2007) study, with two sessions (i.e. 'easy' and 'hard' session) and three set sizes in each session. The tilted lines, which could either be distractors or the target depending on the session, were tilted $17^{\circ}$ clockwise from vertical. Experiment 1, however, only contained one session, in which the target was a vertical line among tilted line distractors. The tilt of the line was smaller, at only $10^{\circ}$ clockwise from vertical, making it more similar to the target. Furthermore, only the set size of 25 elements was included. Finally, it is important to note that the participants in experiment 2 had all but three ( 1 in the ASD and 2 in the control group) participated in experiment 1 , with at least 6 months in between measurements, although the group size was somewhat smaller. In other words, the differences between experiment 1 and 2 are limited to time of measurement, group size and differences in the visual search display (i.e. one versus three set sizes and decreased tilt of lines).

In order to discuss the results of the present experiments in terms of experimental differences (i.e. changes in targetdistractor similarity and the addition of set size as a factor), first we must rule out other possible explanations for the observed effects. As the group of participants in both experiments is highly similar, one could suggest that some form of perceptual learning between sessions has taken place. However, this would suggest that perceptual learning had taken place in the ASD group only, and span at least 6-months. Research on perceptual learning in individuals with ASD show that controls outperform the group diagnosed with ASD on a perceptual learning task they have previously been exposed to (Plaisted et al. 1998b). This would then suggest that, if perceptual learning had taken place, the control group would outperform the ASD group in the current study, which was not the case and is thus not likely to explain the current results. Another possibility is that there is something special about the clinical group in the current study. So far, two studies have previously reported no superior performance in a visual search task for individuals with ASD. First (Constable et al. 2010) find no differences in a visual search task requiring participants to find an ellipse in a set of circles in reaction 
time or error rates between the clinical group and the control group. They suggest this might be due to an older age group compared to previous studies, which would indicate perceptual differences between the clinical group and typically developing individuals could diminish over time (Constable et al. 2010). However, as the age of participants in the present study resembles that of previous research (Kemner et al. 2007; O'Riordan 2004), and superior performance was actually found in experiment 2 (similar to Kemner et al. 2007), this suggestion is not applicable to the present study. Second, Baldassi et al. (2009) find no superiority for locating a deviant stimulus at the center of vision for a group of children with ASD compared to typically developing children. However, as the task in Baldassi et al. (2009) measures sensitivity thresholds for briefly presented sets of stimuli, it is hard to compare their results to the present study. The remaining option is that the difference in results between experiments 1 and 2 is due to differences in the visual search task itself.

As previously stated, the visual search task in experiment 1 differed from that in experiment 2 on a number of factors: only one session was included (i.e. vertical target among tilted distractors), one set size (i.e. 25 elements) was presented, and the distractors were more similar to the target (tilted only $10^{\circ}$ clockwise from vertical instead of $17^{\circ}$ ). Whereas the two sessions in experiment 2 were referred to as 'easy' and 'hard', this categorization of difficulty cannot easily be extended to experiment 1 . Increasing difficulty on a specific task will undoubtedly result in slower reaction times, that is, if the error rates remain constant. However, slower reaction times (at constant error rates) do not necessarily reflect a more difficult task if the task is not identical. The fact that the number of elements in the visual search display, or set size, was not varied in experiment 1 , meant that switching between different set sizes was not necessary. This, in itself, makes the task in experiment 1 different from the task in experiment 2 , and makes comparison of task difficulty troublesome.

Something can, however, be said about the perceptual load of the visual search displays in both tasks, instead of task difficulty. Lavie and Fockert (2003) provide a clear distinction of task difficulty and perceptual load, and the two should not be confused when interpreting the following explanation. As the name implies, perceptual load is the concept of how much load is being placed on the perceptual system, something that increases as both the number of elements in a visual search display is increased as well as when target-distractor similarity is increased (Lavie 1995; Lavie and Cox 1997). This concept of perceptual load provides the basis for load theory, which posits that human perception has limited capacity and automatically uses that capacity for perceptual processing (Lavie 2005; Lavie et al. 2004). If perceptual load on a task is low such that the task does not require all capacity, any capacity remaining is automatically used for task-irrelevant information. When perceptual load on a task in high, no capacity is left, and task-irrelevant information is not processed. This is demonstrated by the decreased interference of distractors when perceptual load is high (Lavie 1995; Lavie and Cox 1997). What this means for the current experiments is that perceptual load increases when set size increases, which is what happens in experiment 2. Furthermore, perceptual load also increases when target-distractor similarity is increased; thus the visual search display in experiment 1 has a higher perceptual load than the largest (i.e. 25 elements) visual search display in experiment 2.

A recent study by Remington et al. (2012) suggests that high perceptual load impairs visual detection in typically developing adults, but not in adults diagnosed with autism. They suggest that superior performance typically observed for individuals with ASD in visual search (Joseph et al. 2009; Kemner et al. 2007; O'Riordan 2004; O'Riordan et al. 2001; Plaisted et al. 1998a) is related to an enhanced perceptual capacity in individuals with ASD. This enhanced capacity implies that individuals with ASD process more information, and although that additional information can be task-irrelevant, it can also be task-relevant (Remington et al. 2012). In this case, the task-relevant information would mean the detection of a target among distractors. Previously, Remington et al. (2009) demonstrated that individuals with ASD require higher levels of perceptual load to be able to ignore irrelevant distractors, again suggesting enhanced capacity for individuals with ASD. Furthermore, this enhanced capacity seems to be reflected in the severity of autistic traits, as typically developing individuals scoring high on the AQ are affected less by higher perceptual load than individuals scoring low on the AQ (Bayliss and Kritikos 2010). While this concept of enhanced perceptual capacity has been used to explain superior performance in individuals with ASD, it might also explain the results observed in experiment 1 of the present study. A possible explanation for the results in the present study is that the perceptual load of the display in experiment 1 has become so high that also the capacity of the individuals with ASD has left no room for the processing of additional task-(ir)relevant information. As such, the critical factor is no longer in capacity for processing multiple information sources, but in the discrimination of a single stimulus-element. With reference to the search displays in the present study this would be mean that the task in experiment 1 is so demanding that both participants with ASD and controls make a decision of target or distractor for one element at a time. In cases of lower perceptual load, as in experiment 2, multiple stimulus-elements can be processed at the same time, with the enhanced perceptual capacity of individuals with ASD allowing for more stimulus-elements to be processed simultaneously. 
If perceptual capacity for both the ASD and control group leaves no room for processing more than one stimulus-element at a time, any superiority for either group would be due to static stimulus discrimination or identification. Previous research has demonstrated that there is no clear evidence for superior or inferior performance in static stimulus discrimination in ASD, which might contribute to the result in the present study (Simmons et al. 2009). For instance, De Jonge et al. (2007) found no differences in contrast sensitivity and thresholds for form discrimination between individuals with ASD and controls. Although Bertone et al. (2005) demonstrated lower thresholds for orientation detection (i.e. detecting whether a stimulus is oriented one way or another) in individuals with ASD under certain circumstances, no differences have been reported on orientation discrimination (i.e. discriminating the tilt of a line from another) between individuals with ASD and controls. This suggests that when the task breaks down to single stimulus-element discrimination, no differences are to be expected, and could explain the results of experiment 1 in the present study.

With reference to the previously noted study of Constable et al. (2010), this could mean that the absence of a difference between individuals with ASD and matchedcontrols in their visual search task might not be due to an older age group, but to limits on perceptual load and the consequent stimulus discrimination. Whether perceptual load in the task Constable et al. (2010) use (i.e. finding an ellipse in a set of distractor circles) is actually of higher perceptual load than finding a tilted line among vertical line distractors has thus far not been tested directly. Future research should prove useful in determining perceptual load across different paradigms. It should be noted, however, that the explanation Constable et al. (2010) provide for their results might still very well be true-that perceptual differences between individuals with ASD and controls can diminish with age.

When the findings of the present study are put in the context of load theory we can hypothesize the following; as long as perceptual capacity leaves room for processing of additional task-(ir)relevant information, individuals with ASD will show superior performance. When perceptual load is so high that no room is left for processing additional task-(ir)relevant information, even in individuals with ASD, no differences will be found on static stimuli between individuals with ASD and controls. Future research will be helpful in testing these claims, pinpointing the scope and limits of the superiority in visual search for individuals with ASD, and uncovering the specific anomalies of visual processing in ASD.

A final note on the results of experiment 2 in the present study is in order. Although the results from the 'easy' session in experiment 2 showed superiority in reaction times for the group diagnosed with ASD compared to controls, this effect was not found in the 'hard' session. Numerically, the effect does appear to be present, even larger than in the 'easy' session, though the effects are not significant. A possible explanation for the lack of statistical significance is the large standard errors in the control group in the 'hard' condition. When we compare the present results with the Kemner et al. (2007) study, whose experiment most closely resembles experiment 2 in the present study, similar standard errors are observed (i.e. around $200 \mathrm{~ms}$ in the larger set sizes when the target is absent). Mean reaction times for the ASD group in the fastest conditions (i.e. 'easy' session when the target is present) are around $600 \mathrm{~ms}$ in both studies, although mean reaction times for the control group in the slowest conditions varyaround $1350 \mathrm{~ms}$ in the present study compared to 1,600 in the Kemner et al. (2007) study. The absence of a significant difference might then be due to a more condensed spread in the reaction times, though the standard errors are quite similar. Furthermore, as the AQ scores in the control population are quite high, this might have lessened the difference between the ASD group and the control group (see e.g. Bayliss and Kritikos 2010). Nevertheless, these considerations do not undermine the finding of superiority in visual search for individuals diagnosed with ASD, as reflected in the 'easy' session. As such, the absence of statistical significance in the 'hard' session does not deter from the conclusions of the present study and the hypothesis regarding perceptual load as a limiting factor for visual search superiority in ASD.

Acknowledgments We thank Branka Milivojevic, Emmie van Schaffelaar, Manje Brinkhuis, Carlijn van den Boomen, and Esther Eijlers for their help with participant recruitment and data collection, and Siarhei Uzunbajakau for building the experimental setup. This work was supported by a Netherlands Organization for Scientific Research (NWO) VICI Grant (45307004) to Chantal Kemner.

Conflict of interest The authors declare that they have no conflict of interest.

\section{References}

Almeida, R. A., Dickinson, J. E., Maybery, M. T., Badcock, J. C., \& Badcock, D. R. (2010). A new step towards understanding Embedded Figures Test performance in the autism spectrum: The radial frequency search task. Neuropsychologia, 48(2), 374-381. doi:10.1016/j.neuropsychologia.2009.09.024.

Bach, M. (1996). The Freiburg Visual Acuity Test-automatic measurement of visual acuity. Optometry and Vision Science, 73(1), 49.

Baldassi, S., Pei, F., Megna, N., Recupero, G., Viespoli, M., Igliozzi, R., et al. (2009). Search superiority in autism within, but not outside the crowding regime. Vision Research, 49(16), 2151-2156. doi:10.1016/j.visres.2009.06.007. 
Baron-Cohen, S., Wheelwright, S., Skinner, R., Martin, J., \& Clubley, E. (2001). The autism-spectrum quotient (AQ): Evidence from asperger syndrome/high-functioning autism, malesand females, scientists and mathematicians. Journal of Autism and Developmental Disorders, 31(1), 5-17. doi:10.1023/A:1005653411471.

Bayliss, A. P., \& Kritikos, A. (2010). Brief report: Perceptual load and the autism spectrum in typically developed individuals. Journal of Autism and Developmental Disorders, 41(11), 1573-1578. doi:10.1007/s10803-010-1159-8.

Bertone, A., Mottron, L., Jelenic, P., \& Faubert, J. (2005). Enhanced and diminished visuo-spatial information processing in autism depends on stimulus complexity. Brain, 128, 2430-2441. doi: 10.1093/brain/awh561.

Constable, P. A., Solomon, J. A., Gaigg, S. B., \& Bowler, D. M. (2010). Crowding and visual search in high functioning adults with autism spectrum disorder. Clinical Optometry, 93. doi: 10.2147/OPTO.S11476.

Dakin, S., \& Frith, U. (2005). Vagaries of visual perception in autism. Neuron, 48(3), 497-507. doi:10.1016/j.neuron.2005.10.018.

De Jonge, M. V., Kemner, C., de Haan, E. H., Coppens, J. E., van den Berg, T. J. T. P., \& van Engeland, H. (2007). Visual information processing in high-functioning individuals with autism spectrum disorders and their parents. Neuropsychology, 21(1), 65-73. doi: 10.1037/0894-4105.21.1.65.

Elsabbagh, M., Volein, A., Csibra, G., Holmboe, K., Garwood, H., Tucker, L., et al. (2009). Neural correlates of eye gaze processing in the infant broader autism phenotype. Biological Psychiatry, 65(1), 31-38. doi:10.1016/j.biopsych.2008.09.034.

Hooge, I. T. C., \& Erkelens, C. J. (1999). Peripheral vision and oculomotor control during visual search. Vision Research, 39(8), 1567-1575. doi:10.1016/S0042-6989(98)00213-2.

Jarrold, C., Gilchrist, I. D., \& Bender, A. (2005). Embedded figures detection in autism and typical development: Preliminary evidence of a double dissociation in relationships with visual search. Developmental Science, 8(4), 344-351. doi:10.1111/ j.1467-7687.2005.00422.x.

Joseph, R. M., Keehn, B., Connolly, C., Wolfe, J. M., \& Horowitz, T. S. (2009). Why is visual search superior in autism spectrum disorder? Developmental Science, 12(6), 1083-1096. doi: 10.1111/j.1467-7687.2009.00855.x.

Kaldy, Z., Kraper, C., Carter, A. S., \& Blaser, E. (2011). Toddlers with autism spectrum disorder are more successful at visual search than typically developing toddlers. Developmental Science, 14(5), 980-988. doi:10.1111/j.1467-7687.2011.01053.x.

Kemner, C., Ewijk, L., Engeland, H., \& Hooge, I. (2007). Brief report: Eye movements during visual search tasks indicate enhanced stimulus discriminability in subjects with PDD. Journal of Autism and Developmental Disorders, 38(3), 553-557. doi:10.1007/s10803-007-0406-0.

Lavie, N. (1995). Perceptual load as a necessary condition for selective attention. Journal of Experimental Psychology: Human Perception and Performance, 21(3), 451-468. doi:10.1037/ 0096-1523.21.3.451

Lavie, N. (2005). Distracted and confused?: Selective attention under load. Trends in Cognitive Sciences, 9(2), 75-82. doi:10.1016/ j.tics.2004.12.004.

Lavie, N., \& Cox, S. (1997). On the efficiency of visual selective attention: Efficient visual search leads to inefficient distractor rejection. Psychological Science, 8(5), 395-396. doi:10.1111/ j.1467-9280.1997.tb00432.x.

Lavie, N., \& Fockert, J. W. (2003). Contrasting effects of sensory limits and capacity limits in visual selective attention. Perception \& Psychophysics, 65(2), 202-212. doi:10.3758/BF03194795.

Lavie, N., Hirst, A., de Fockert, J. W., \& Viding, E. (2004). Load theory of selective attention and cognitive control. Journal of Experimental Psychology: General, 133(3), 339-354. doi: 10.1037/0096-3445.133.3.339.

Lord, C., Rutter, M., \& Couteur, A. (1994). Autism diagnostic interview-revised: A revised version of a diagnostic interview for caregivers of individuals with possible pervasive developmental disorders. Journal of Autism and Developmental Disorders, 24(5), 659-685. doi:10.1007/BF02172145.

Lord, C., Rutter, M., Goode, S., Heemsbergen, J., Jordan, H., Mawhood, L., et al. (1989). Autism diagnostic observation schedule: A standardized observation of communicative and social behavior. Journal of Autism and Developmental Disorders, 19(2), 185-212. doi:10.1007/BF02211841.

Merin, N., Young, G. S., Ozonoff, S., \& Rogers, S. J. (2006). Visual fixation patterns during reciprocal social interaction distinguish a subgroup of 6-month-old infants at-risk for autism from comparison infants. Journal of Autism and Developmental Disorders, 37(1), 108-121. doi:10.1007/s10803-006-0342-4.

O'Riordan, M. A. (2004). Superior visual search in adults with autism. Autism, 8(3), 229-248. doi:10.1177/1362361304045219.

O’Riordan, M. A., Plaisted, K. C., Driver, J., \& Baron-Cohen, S. (2001). Superior visual search in autism. Journal of Experimental Psychology: Human Perception and Performance, 27(3), 719-730. doi:10.1037/0096-1523.27.3.719.

Pellicano, E., Smith, A. D., Cristino, F., Hood, B. M., Briscoe, J., \& Gilchrist, I. D. (2011). Children with autism are neither systematic nor optimal foragers. Proceedings of the National Academy of Sciences, 108(1), 421-426. doi:10.1073/pnas. 1014076108.

Plaisted, K., O’Riordan, M., \& Baron-Cohen, S. (1998a). Enhanced visual search for a conjunctive target in autism: A research note. Journal of Child Psychology and Psychiatry, 39(5), 777-783. doi:10.1111/1469-7610.00376.

Plaisted, K., O'Riordan, M., \& Baron-Cohen, S. (1998b). Enhanced discrimination of novel, highly similar stimuli by adults with autism during a perceptual learning task. Journal of Child Psychology and Psychiatry, 39(5), 765-775. doi:10.1111/14697610.00375 .

Remington, A., Swettenham, J., Campbell, R., \& Coleman, M. (2009). Selective attention and perceptual load in autism spectrum disorder. Psychological Science, 20(11), 1388-1393. doi:10.1111/j.1467-9280.2009.02454.x.

Remington, A. M., Swettenham, J. G., \& Lavie, N. (2012). Lightening the load: Perceptual load impairs visual detection in typical adults but not in autism. Journal of Abnormal Psychology, 121(2), 544-551. doi:10.1037/a0027670.

Simmons, D. R., Robertson, A. E., McKay, L. S., Toal, E., McAleer, P., \& Pollick, F. E. (2009). Vision in autism spectrum disorders. Vision Research, 49(22), 2705-2739. doi:10.1016/j.visres. 2009.08.005. 